\title{
Consumption of sugar sweetened beverage is associated with incidence of metabolic syndrome in Tehranian children and adolescents
}

\author{
Parvin Mirmiran ${ }^{1 *}$, Emad Yuzbashian ${ }^{1}$, Golaleh Asghari ${ }^{1}$, Somayeh Hosseinpour-Niazi ${ }^{1}$ and Fereidoun Azizi ${ }^{2}$
}

\begin{abstract}
Background: Intakes of high sugar-sweetened beverages (SSBs) in adults can escalate risk of metabolic syndrome (MetS); however, data of longitudinal studies in children and adolescents are lacking. In this study we assessed consumption of SSBs in relation to incidence of MetS among children and adolescents during a 3.6 year follow-up.

Methods: This study was a population-based longitudinal study, in which 424 subjects, aged 6-18 years, from the Tehran Lipid and Glucose Study with complete data on dietary intake, blood pressure, anthropometry, and biochemical indices were followed for 3.6 years. Dietary intake was collected using a valid and reliable food frequency questionnaire. MetS was defined according to the Cook criteria. Sugar sweetened beverages included all kinds of sugar sweetened carbonated soft drinks (SSSDs) and fruit juice drinks.

Results: Average daily intakes of SSSD and fruit juice drinks were $38.5 \pm 75.0$ and $32.3 \pm 60.1 \mathrm{~g}$, respectively. After adjustment for confounders, compared to the first quartile, the odds ratio of incident MetS in the highest quartile of SSB and SSSD was 3.20 (95 \% Cl: 1.06-9.90) and 3.01 (95\% Cl: 1.17-7.74), respectively. Regarding incidence of MetS components, compared with the lowest quartile, the highest quartile of SSSDs showed odds ratios of 2.49 (95\% Cl: 1.00-6.53) for abdominal obesity and 2.79 (95 \% Cl: 1.02-7.64) for hypertension. No significant association was found between consumption of fruit juice drink and SSSD with other components of MetS.
\end{abstract}

Conclusions: Children and adolescents with high intakes of carbonated beverages could be at increased risk of MetS, abdominal obesity, and hypertension.

Keywords: Metabolic syndrome, Children, Adolescents, Sugar sweetened carbonated soft drink, Fruit juice drink, Sugar sweetened beverage

\section{Introduction}

The rising prevalence of metabolic syndrome (MetS) during childhood and adolescence affectes over $3.3 \%$ of the adolescent population worldwide [1], and may be predictive of adult MetS, type 2 diabetes, and cardiovascular disease $[2,3]$. Although some studies have examined genetic predispositions, findings show that only $10 \%$ of MetS cases can be explained by genetics [4]. Alternatively, many environmental factors have been identified [5], e.g. food intakes play an important role in the

\footnotetext{
* Correspondence: mirmiran@endocrine.ac.ir

${ }^{1}$ Nutrition and Endocrine Research Center, Research Institute for Endocrine Sciences, Shahid Beheshti University of Medical Sciences, Tehran, Iran

Full list of author information is available at the end of the article
}

pathogenesis of MetS through hepatic insulin resistance and/or increasing reactive oxygen species formation [6].

Nowadays the consumption of sugar sweetened beverages (SSBs) has increased drastically particularly in children and adolescents [7-9]. Higher SSB intakes in adults may enhance risk of weight gain and of developing obesity and obesity-related disorders, such as MetS, type 2 diabetes, coronary heart disease, and stroke $[10,11]$. However, according to a recent evidence mapping study by Althuis et al. [12], longitudinal studies in children and adolescents are lacking and limited to one crosssectional investigation, which indicated that SSBs have been linked to increased risk of MetS, hypertension, and abdominal obesity [13].

\section{Biomed Central}

(C) 2015 Mirmiran et al. This is an Open Access article distributed under the terms of the Creative Commons Attribution License (http://creativecommons.org/licenses/by/4.0), which permits unrestricted use, distribution, and reproduction in any medium, provided the original work is properly credited. The Creative Commons Public Domain Dedication waiver (http:// creativecommons.org/publicdomain/zero/1.0/) applies to the data made available in this article, unless otherwise stated. 
In the current study, we evaluated the association between SSB consumption with incident MetS and its components 3.6 years later among children and adolescents in Iran.

\section{Materials and method}

Tehran Lipid and Glucose Study (TLGS) is a prospective community-based study being conducted to determine the risk factors of and prevent non-communicable diseases by implementing and promoting healthy lifestyles [14].

Baseline data were collected from 15005 participants, aged 3 years and over, of residents District No. 13 of Tehran under coverage of three medical health centers. The participants were followed up every 3 years to update their data on demographic, lifestyle, biochemical, clinical, and dietary measurements; the baseline survey was a cross-sectional study conducted from 1999 to 2001, and surveys 2 (2002-2005), 3 (2006-2008), and 4 (2009-2011) were prospective follow-up surveys.

In the third survey of the TLGS (2006-2008), from among 12523 examined participants, 3462 were randomly selected for dietary assessment; of these, 621 individuals were aged 6-18 y who included in the current study. Participants who had incomplete dietary intakes or had missing measures of MetS components $(n=29)$ were excluded. Additionally, participants with reported energy intakes $(\mathrm{kcal} /$ day) to energy requirements ratios beyond \pm 3 SD range, were also excluded $(n=6)$ because they fell into the over-or under-reporting categories [15]. Participants were followed until survey 4 (2009-2011), an average of 3.6 years (follow-up rate: $86 \%$ ). Depending on the outcome variable under analysis, we excluded persons whose data at survey $3(2006-2008)$ indicated prevalent MetS $(n=69)$, hypertension $(n=53)$, high triglycerides (TGs) $(n=168)$, low high density lipoprotein cholesterol (HDL-C) $(n=242)$, high fasting plasma glucose (FPG) $(n=12)$, or abdominal obesity $(n=145)$; some individuals fell into more than one exclusion category. The final sample sizes in fully adjusted models varied by outcomes as follows: MetS $(n=424)$, abdominal obesity $(n=327)$, high triglycerides (TGs) $(n=347)$, hypertension $(n=439)$, low HDL-C $(n=290)$, and high fasting plasma glucose $(n=476)$.

The design of this study was approved by the institutional ethics committee of the Research Institute for Endocrine Sciences, Shahid Beheshti University of Medical Sciences, and written informed consent was obtained from participants' parents.

\section{Dietary intake}

A valid and reliable 168-item semi-quantitative food frequency questionnaire (FFQ) [16-18] was used by trained dietitians, during face-to face interviews, to evaluate the usual dietary intakes of participants. Participants were asked to report their consumption frequency of food items during the previous year on a daily, weekly, or monthly basis. Mothers were asked about the type and quantity of meals and snacks when children were unable to recall. Portion sizes of consumed foods were specified according to the US Department of Agriculture (USDA) standard portion sizes or household measures (e.g. beans, 1 tablespoon; chicken meat, 1 leg or wing; rice, 1 large or small plate) [19]. Obtained data were converted to mean daily intake in grams or milliliters. This FFQ included questions on the frequency of consumption and usual portion size of all kinds of sugar sweetened carbonated soft drinks (SSSDs) and fruit juice drinks (both $100 \%$ fruit juice and sugar sweetened synthetic juice drinks that are not $100 \%$ juice). According to Althuis et al. [12], these items were combined to estimate the daily intake of SSBs $(\mathrm{ml} / \mathrm{d})$. SSB intakes were divided by using quartile cutoffs, with the assumption that one serving was equivalent to 1 cup $(250 \mathrm{~mL})$.

To study the reproducibility of the FFQ, 132 subjects (61 men and 71 women) twice completed a 168-item FFQ (FFQ1, FFQ2), with a 14-month interval between FFQ1 and FFQ2, and to assess the validity, 12 dietary recalls (DRs) were collected (1 each month) over the 1year interval. The age-and energy-adjusted and deattenuated Spearman correlation coefficients used to assess validity of the FFQ for soft drinks were 0.54 and 0.37 in men and women, respectively. The age-and energyadjusted intraclass correlation coefficients, which reflect the reproducibility of food groups in the FFQ, were 0.61 and 0.65 in men and in women, respectively [17].

\section{Measurements}

Information about physical activity was collected using the modifiable activity questionnaire (MAQ) to calculate metabolic equivalent task (MET) minutes per week [20]. High reliability $(98 \%)$ and moderate validity $(47 \%)$ were ascertained for the MAQ translated into Persian [21]. Levels of physical activity were categorized as light (MET <600 min/wk), moderate (MET 600-1,499 min/ wk), and vigorous (MET $\geq 1,500 \mathrm{~min} / \mathrm{wk}$ ).

Weight was measured, while participants were minimally clothed without shoes, using digital scales (Seca 707, Seca Corp., Hanover, MD; range 0.1-150 kg) and recorded to the nearest $100 \mathrm{~g}$. Height was measured in a standing position without shoes, using a stadiometer, with shoulders in normal alignment. Body mass index (BMI) was calculated as weight (kg) divided by the square of height $\left(\mathrm{m}^{2}\right)$. Waist circumference (WC) was measured at the umbilicus, using a measuring tape, without pressure to body surfaces, and was recorded to the nearest $0.5 \mathrm{~cm}$.

Blood pressure was measured twice, after participants were seated for $15 \mathrm{~min}$, using a standard mercury 
sphygmomanometer; there was at least a $30-\mathrm{s}$ interval between these two separate measurements, and the mean of two measurements was considered the patient's blood pressure.

Blood samples, at baseline and follow up, were drawn between 7:00 and 9:00 AM from all study participants after 12-14 h overnight fasting; all blood analyses were done at the TLGS research laboratory on the day of blood collection. FPG was measured by the enzymatic colorimetric method using glucose oxidase. Serum HDL$\mathrm{C}$ was measured after precipitation of the apolipoprotein B-containing lipoproteins with phosphotungstic acid and serum TGs were assayed using an enzymatic colorimetric method with glycerol phosphate oxidase. These analyses were performed using commercial kits (Pars Azmoon Inc., Tehran, Iran) and a Selectra 2 auto analyzer (Vital Scientific, Spankeren, The Netherlands). Inter-and intra-assay coefficients of variations at baseline were both $2.2 \%$ for FPG, 2 and $0.5 \%$ for HDL-C and 1.6 and $0.6 \%$ for TGs, respectively.

\section{Definitions}

In children and adolescents, MetS was defined according to definition proposed by Cook et al. as 3 or more of the following [22]: Fasting TGs $\geq 110 \mathrm{mg} / \mathrm{dl}$; HDL-C $<$ $40 \mathrm{mg} / \mathrm{dl} ; \mathrm{WC} \geq 90$ th percentile for age and sex, according to national reference curves [23]; systolic blood pressure and diastolic blood pressure $\geq 90$ th percentile for sex, age and height, from the National Heart, Lung, and Blood Institute's recommended cut-off points [24], and FPG $\geq 100 \mathrm{mg} / \mathrm{dl}$, according to the recommendations of American Diabetes Association [25]. In subjects aged > 18 years after follow-up, MetS was defined according to the joint interim statement [26] as the presence of any 3 of 5 risk factors of the following: 1) Abdominal obesity as $\mathrm{WC} \geq 91$ and men $\geq 89 \mathrm{~cm}$ for women and men, respectively, according to Iranians cut-off points [27]; 2) FPG $\geq 100 \mathrm{mg} / \mathrm{dl}$ or drug treatment; 3) fasting TGs $\geq 150 \mathrm{mg} / \mathrm{dl}$ or drug treatment; 4) fasting HDL-C $<50 \mathrm{mg} / \mathrm{dl}$ for women and $<40 \mathrm{mg} / \mathrm{dl}$ for men or drug treatment, and 5) hypertension was defined as systolic blood pressure $\geq 130 \mathrm{mmHg}$, diastolic blood pressure $\geq 85 \mathrm{mmHg}$, or antihypertensive drug treatment.

\section{Statistical analysis}

All analyses were conducted using the Statistical Package for Social Sciences (version 15.0; SPSS Inc, Chicago IL). Baseline characteristics of subjects were expressed as mean \pm SD or median (interquartile 25-75) for continuous variables and percentages for categorical variables. To investigate the trend of continuous and categorical variables according to quartiles of SSB, linear regression and chi-square test were used, respectively. There were no significant differences in baseline anthropometric and biochemical assessments between subjects who provided follow-up assessments and those lost to follow-up (data not shown).

Intakes of $\mathrm{SSBs} \quad(<19.2, \quad 19.2-43.8, \quad 43.9-91.5$, and $>91.6 \mathrm{ml} /$ day), SSSDs $(<7.6,7.6-15.3,15.3-40.0$, and $>40.0 \mathrm{ml} /$ day $)$, and fruit juice drinks $(<4.1,4.1-13.5$, $13.5-34.9$, and $34.9 \mathrm{ml} /$ day) were categorized into the quartile cut off points. To estimate the risk of 3.6-year incident outcomes, separate multiple regression models were used for abdominal obesity, high FPG, high TGs, low HDL-C, hypertension, and MetS with quartiles of SSB, SSSD, and fruit juice drink consumption; odds ratios (OR) and $95 \%$ confidence interval (CI) were reported. The initial model was adjusted for age, sex, total energy intake, physical activity, and family history of diabetes. Further adjustment for dietary fiber, tea and coffee, red and processed meat, fruits, and vegetables was made and the final model was additionally adjusted for BMI.

\section{Results}

The mean \pm SD age of study population (68 \% girls) was $13.6 \pm 3.7$ years; the mean daily SSB intake was $98 \mathrm{ml}$ in boys and $70 \mathrm{ml}$ in girls. After a 3.6 year follow up, cumulative incidence of MetS was $11 \%$.

The descriptive characteristics of the study population according to quartile of SSB consumption are shown in Table 1. Participants with higher SSB consumption were likely to have higher systolic blood pressure, FPG, and BMI; in addition, subjects with higher SSB consumption had higher intakes of daily energy, saturated fatty acids, trans-fatty acids, glucose, fructose, sucrose, simple sugars, higher glycemic index, and lower intakes of fruits and whole grains.

Sugar sweetened beverage intake was inversely related to MetS and its components after adjustment for potential confounders (Table 2). Further adjustment for dietary factors did not have a major impact on the results. In this model, subjects in the highest quartile of SSB consumption had increased risk of incident MetS (OR: 3.16; $95 \%$ CI: $1.02-9.78, P$ for trend $=0.007$ ) and abdominal obesity (OR: 2.97; $95 \%$ CI: $1.23-7.19, P$ for trend $=0.017$ ). Adjustment for BMI slightly strengthened the association; adjusted multivariable ORs of MetS, abdominal obesity, and hypertension were $3.50,3.66$, and 2.90 (all $P$ for trends $<0.05$ ).

Higher intakes of SSSD was associated with significant increase in the risk of incident MetS (OR: 3.58, $95 \%$ CI: $1.09-11.78, P$ for trend $=0.013)$, abdominal obesity (OR: $3.78,95 \%$ CI: $1.08-13.27, P$ for trend $=$ 0.043), and hypertension (OR: 2.74, 95 \% CI: 1.05-7.19, $P$ for trend $=0.018$ ) in the fully adjusted model. Associations for fruit juice drink intake were not statistically significant (Table 3). 
Table 1 Baseline characteristics of children and adolescents according to quartiles of sugar sweetened beverage consumption: Tehran Lipid and Glucose Study

\begin{tabular}{|c|c|c|c|c|c|}
\hline & \multicolumn{4}{|l|}{ Quartiles } & \multirow{2}{*}{$\begin{array}{l}P \text { for } \\
\text { trend' }\end{array}$} \\
\hline & $\mathrm{Q} 1(n=106)$ & Q2 $(n=106)$ & Q3 $(n=106)$ & $\mathrm{Q} 4(n=106)$ & \\
\hline Median intake (ml/d) & 9.3 & 32.0 & 58.6 & 142.2 & \\
\hline Age (years) & $13.8 \pm 3.7$ & $12.9 \pm 3.7$ & $13.8 \pm 3.7$ & $13.7 \pm 3.6$ & 0.737 \\
\hline Girls (\%) & 72 & 43 & 62 & 50 & 0.092 \\
\hline Systolic blood pressure $(\mathrm{mmHg})$ & $96.8 \pm 12.2$ & $97.7 \pm 10.7$ & $97.1 \pm 11.7$ & $100.2 \pm 12.0$ & 0.021 \\
\hline Diastolic blood pressure $(\mathrm{mmHg})$ & $64.3 \pm 9.4$ & $63.9 \pm 6.9$ & $65.7 \pm 9.1$ & $64.7 \pm 10.2$ & 0.520 \\
\hline Triglycerides (mg/dl) & $83.0(62.7-103.2)$ & $77.0(63.0-94.0)$ & $84.0(64.0-105.2)$ & $81.5(63.0-112.5)$ & 0.216 \\
\hline Fasting plasma glucose (mg/dl) & $83.9 \pm 5.9$ & $84.4 \pm 5.9$ & $85.5 \pm 5.8$ & $86.0 \pm 6.5$ & 0.037 \\
\hline High density lipoprotein (mg/dl) & $45.8 \pm 11.9$ & $46.3 \pm 11.11$ & $47.3 \pm 9.6$ & $43.8 \pm 9.4 .2$ & 0.269 \\
\hline Waist circumstance (cm) & $69.7 \pm 11.1$ & $69.7 \pm 10.4$ & $67.8 \pm 10.6$ & $68.1 \pm 9.5$ & 0.231 \\
\hline Body mass index $\left(\mathrm{kg} / \mathrm{m}^{2}\right)$ & $18.7 \pm 4.3$ & $19.2 \pm 3.8$ & $19.7 \pm 3.6$ & $20.1 \pm 3.8$ & $<0.001$ \\
\hline \multicolumn{6}{|l|}{ Daily dietary intakes } \\
\hline Total energy (kcal) & $2116 \pm 962$ & $2364 \pm 1017$ & $2440 \pm 1020$ & $3317 \pm 1670$ & $<0.001$ \\
\hline Dietary fiber/1000 kcal (g) & $14.5 \pm 6.4$ & $14.4 \pm 6.6$ & $14.6 \pm 5.5$ & $14.2 \pm 4.6$ & 0.866 \\
\hline Total fat (\% energy) & $32.4 \pm 8.5$ & $31.9 \pm 6.3$ & $32.3 \pm 7.2$ & $31.6 \pm 5.5$ & 0.785 \\
\hline Trans-fatty acids (g) & $4.17 \pm 3.88$ & $4.23 \pm 2.83$ & $5.26 \pm 5.77$ & $6.6 \pm 4.6$ & $<0.001$ \\
\hline Saturated fatty acids (g) & $25.1 \pm 12.7$ & $28.1 \pm 13.2$ & $27.6 \pm 16.4$ & $41.9 \pm 23.2$ & $<0.001$ \\
\hline Protein (\% energy) & $13.0 \pm 2.4$ & $13.8 \pm 3.4$ & $12.8 \pm 1.9$ & $12.9 \pm 1.9$ & 0.230 \\
\hline Carbohydrate (\% energy) & $56.2 \pm 8.7$ & $56.8 \pm 7.4$ & $57.1 \pm 7.1$ & $57.5 \pm 5.9$ & 0.098 \\
\hline Glucose (g) & $11.7 \pm 7.8$ & $14.7 \pm 7.5$ & $15.2 \pm 7.2$ & $25.2 \pm 15.2$ & $<0.001$ \\
\hline Fructose (g) & $13.2 \pm 8.8$ & $16.5 \pm 8.2$ & $18.2 \pm 8.7$ & $23.9 \pm 7.9$ & $<0.001$ \\
\hline Sucrose (g) & $26.8 \pm 21.2$ & $30.6 \pm 20.3$ & $36.5 \pm 60.4$ & $41.1 \pm 28.4$ & $<0.001$ \\
\hline Total simple sugar (g) & $111.4 \pm 81.8$ & $118.7 \pm 56.0$ & $124.3 \pm 81.9$ & $142.9 \pm 98.0$ & $<0.001$ \\
\hline Total simple sugar (\% energy) & $20.8 \pm 7.7$ & $20.3 \pm 5.2$ & $20.2 \pm 5.76$ & $21.6 \pm 5.7$ & 0.422 \\
\hline Glycemic index & $61.1 \pm 35.8$ & $78.4 \pm 38.2$ & $81.5 \pm 45.11$ & $102.1 \pm 74.5$ & $<0.001$ \\
\hline Fruit (g/1000 kcal) & $183.4 \pm 118$ & $173.5 \pm 113.6$ & $160.5 \pm 94.4$ & $130.4 \pm 100.1$ & $<0.001$ \\
\hline Vegetable (g/1000 kcal) & $105.7 \pm 66.2$ & $94.8 \pm 55.8$ & $97.4 \pm 53.5$ & $94.5 \pm 46.2$ & 0.200 \\
\hline Low fat dairy (g/1000 kcal) & $117.9 \pm 90.3$ & $113.9 \pm 89.7$ & $109.1 \pm 75.3$ & $121.5 \pm 91.9$ & 0.875 \\
\hline Meat and processed meat (g/1000 kcal) & $11.4 \pm 11.9$ & $12.9 \pm 9.4$ & $12.5 \pm 10.1$ & $14.9 \pm 11.2$ & 0.076 \\
\hline Legumes (g/1000 kcal) & $7.0 \pm 8.9$ & $6.9 \pm 7.6$ & $7.3 \pm 9.1$ & $5.9 \pm 7.1$ & 0.362 \\
\hline Whole grains (g/1000 kcal) & $38.7 \pm 40.9$ & $30.9 \pm 31.6$ & $27.0 \pm 25.0$ & $26.5 \pm 26.6$ & 0.003 \\
\hline
\end{tabular}

Data represented as mean \pm SD or median (IQ 25-75) for continuous variables and percentage for categorically distributed variables

${ }^{1}$ Based on linear regression models for continuous variables and chi-square for categorical variables

\section{Discussion}

In the present study, we provided evidence that children and adolescents who consumed SSB over $90 \mathrm{ml} /$ day (one third of a serving) had almost a 3-fold increased risk of incident MetS, abdominal obesity, and hypertension independent of confounders, particularly BMI. Similarly, higher SSSD consumption was directly associated with incidence of MetS, abdominal obesity, and hypertension.

Findings of the current study support those of prospective ones conducted on SSBs and MetS in adults $[28,29]$, which report that adults with higher SSB consumption had a $0.5-2$ fold increased risk of incident MetS. To our knowledge, the present study is the first to examine the association of SSB, SSSD, and fruit juice drink consumption with MetS in longitudinal analysis in children and adolescents. Our findings are consistent with previously published cross-sectional reports in which high SSBs consumption was associated with prevalent MetS and higher insulin resistance in children and adolescents [13, 30, 31]. Adolescents, aged 12 to 16 years, with higher consumption of SSB had a 5.1-fold increased prevalence of MetS [13]. Also each additional SSB serving intake was associated with a $5 \%$ increment 
Table 2 Multivariable-adjusted ORs (95\% Cls) of incident MetS and its components by quartiles of sugar sweetened beverage consumption after 3.6 years of follow up among children and adolescents

\begin{tabular}{|c|c|c|c|c|c|}
\hline & \multicolumn{4}{|c|}{ Quartiles } & \multirow{2}{*}{$\begin{array}{l}P \text { for } \\
\text { trend }\end{array}$} \\
\hline & $\mathrm{Q} 1$ & Q2 & Q3 & Q4 & \\
\hline Median intake $(\mathrm{ml} / \mathrm{d})$ & 9.3 & 32.0 & 58.6 & 142.2 & \\
\hline \multicolumn{6}{|l|}{ MetS } \\
\hline Model 1 & 1.00 & $1.06(0.31-3.57)$ & $2.84(0.97-8.37)$ & $3.00(0.99-9.05)$ & 0.009 \\
\hline Model 2 & 1.00 & $1.02(0.30-3.48)$ & $2.94(0.99-8.74)$ & $3.16(1.02-9.78)$ & 0.007 \\
\hline Model 3 & 1.00 & $1.11(0.32-3.82)$ & $2.96(0.99-8.80)$ & $3.50(1.12-10.92)$ & 0.005 \\
\hline \multicolumn{6}{|l|}{ Low HDL-C } \\
\hline Model 1 & 1.00 & $0.72(0.24-2.16)$ & $0.96(0.33-2.82)$ & $0.55(0.17-1.81)$ & 0.434 \\
\hline Model 2 & 1.00 & $0.61(0.19-1.89)$ & $0.93(0.31-2.78)$ & $0.42(0.11-1.55)$ & 0.320 \\
\hline Model 3 & 1.00 & $0.65(0.21-2.07)$ & $0.97(0.32-2.93)$ & $0.45(0.12-1.66)$ & 0.386 \\
\hline \multicolumn{6}{|l|}{ Abdominal obesity } \\
\hline Model 1 & 1.00 & $1.53(0.63-3.71)$ & $1.65(0.61-3.94)$ & $2.94(1.27-6.82)$ & 0.012 \\
\hline Model 2 & 1.00 & $1.58(0.65-3.86)$ & $1.70(0.70-4.09)$ & $2.97(1.23-7.19)$ & 0.017 \\
\hline Model 3 & 1.00 & $2.16(0.82-5.68)$ & $1.86(0.71-4.84)$ & $3.66(1.40-9.59)$ & 0.016 \\
\hline \multicolumn{6}{|l|}{ Hypertension } \\
\hline Model 1 & 1.00 & $1.46(0.45-4.77)$ & $2.66(0.89-7.96)$ & $2.41(0.79-7.73)$ & 0.070 \\
\hline Model 2 & 1.00 & $1.47(0.45-4.82)$ & $2.68(0.89-8.11)$ & $2.45(0.78-7.70)$ & 0.072 \\
\hline Model 3 & 1.00 & $1.73(0.52-5.74)$ & $3.02(0.98-9.25)$ & $2.90(0.91-9.26)$ & 0.043 \\
\hline \multicolumn{6}{|l|}{ High FPG } \\
\hline Model 1 & 1.00 & $1.22(0.56-3.22)$ & $1.90(0.76-4.72)$ & $2.07(0.79-5.39)$ & 0.079 \\
\hline Model 2 & 1.00 & $1.17(0.44-3.08)$ & $1.83(0.73-4.58)$ & $1.90(0.71-5.09)$ & 0.109 \\
\hline Model 3 & 1.00 & $1.21(0.48-3.21)$ & $1.87(0.75-4.68)$ & $1.95(0.73-5.22)$ & 0.108 \\
\hline \multicolumn{6}{|l|}{ High TGs } \\
\hline Model 1 & 1.00 & $0.76(0.24-2.38)$ & $1.57(0.57-4.33)$ & $1.70(0.58-4.99)$ & 0.156 \\
\hline Model 2 & 1.00 & $0.74(0.23-2.33)$ & $1.53(0.55-4.29)$ & $1.66(0.55-5.05)$ & 0.173 \\
\hline Model 3 & 1.00 & $0.82(0.26-2.61)$ & $1.62(0.57-4.58)$ & $1.80(0.59-5.25)$ & 0.148 \\
\hline
\end{tabular}

MetS metabolic syndrome, HDL-C high density lipoprotein-cholesterol, FPG fasting plasma glucose, TGs triglycerides

Model1: Adjusted for baseline age, sex, total energy intake, physical activity, and family history of diabetes

Model 2: Additionally adjusted for dietary fiber, tea and coffee, red and processed meat, fruit, and vegetable

Model 3: Additionally adjusted for body mass index

${ }^{1}$ Based on logistic regression model using median intake of sugar sweetened beverages in each quartile as a continuous variable

of insulin resistance among 12-19 year-old American adolescents [31]. In addition, higher SSB consumption in children, aged 8-10 years, was associated with a 0.1-unit higher HOMA-IR [30]. Our prospective findings confirmed the proposed causal link in cross-sectional studies between SSB consumption, insulin resistance, and MetS.

Sugar sweetened carbonated soft drinks are among the components of SSB and in the current study, had a positive association with incident MetS, abdominal obesity, and hypertension. Similarly, these beverages are associated with greater odds of unfavorable changes in cardiometabolic risk factors, independent of body weight [31, 32]. The Western Australian Cohort (Raine) Study showed that SSB consumption of over 1.3 servings/d, was associated with a 3.2-fold increment of overall cardio-metabolic risk, and had unfavorable effects on changes of waist circumference, TGs, and HDL-C, independent of BMI after a 2-year follow-up [32]. Our results are also consistent with a cross-sectional study of 12-19 year-old American adolescents, who consumed on average 7.4 servings of SSBs daily, indicating that each additional SSB serving intake was associated with increments of systolic blood pressure and waist circumference, and with decreased HDL-C, after adjusting for age, sex, race, menarche, and energy intake, but not for BMI [31]. Interestingly, our study found an approximately 3.6-fold greater risk of MetS following consumption of a one third of serving of SSSDs comparable to results of studies with similar cardio-metabolic risks [32] but with much higher consumption, highlighting the magnitude of this association in the current study. 
Table 3 Multivariable-adjusted ORs (95\% Cls) of incident MetS and its components by quartiles of sugar sweetened carbonated soft drink and fruit juice drink intakes after 3.6 years of follow up among children and adolescents

\begin{tabular}{|c|c|c|c|c|c|}
\hline \multirow[b]{3}{*}{ Sugar sweetened carbonated soft drink $(\mathrm{ml} / \mathrm{d})$} & \multicolumn{4}{|c|}{ Quartiles } & \multirow{3}{*}{$\begin{array}{l}P \text { for } \\
\text { trend }\end{array}$} \\
\hline & Q1 & Q2 & Q3 & Q4 & \\
\hline & 1.12 & 9.33 & 33.06 & 100.00 & \\
\hline \multicolumn{6}{|l|}{ MetS } \\
\hline Model 1 & 1.00 & $1.06(0.31-3.57)$ & $2.29(0.76-6.97)$ & $3.33(1.04-10.73)$ & 0.014 \\
\hline Model 2 & 1.00 & $1.02(0.30-3.47)$ & $2.39(0.78-7.31)$ & $3.45(1.06-11.18)$ & 0.012 \\
\hline Model 3 & 1.00 & $1.11(0.32-3.82)$ & $2.44(0.79-7.49)$ & $3.58(1.09-11.78)$ & 0.013 \\
\hline \multicolumn{6}{|l|}{ Low HDL-C } \\
\hline Model 1 & 1.00 & $0.38(0.12-1.30)$ & $0.77(0.31-1.93)$ & $1.00(0.33-2.98)$ & 0.982 \\
\hline Model 2 & 1.00 & $0.37(0.11-1.27)$ & $0.71(0.28-1.84)$ & $1.15(0.35-3.79)$ & 0.950 \\
\hline Model 3 & 1.00 & $0.37(0.11-1.28)$ & $0.69(0.26-1.80)$ & $1.15(0.35-3.81)$ & 0.989 \\
\hline \multicolumn{6}{|l|}{ Abdominal obesity } \\
\hline Model 1 & 1.00 & $1.73(0.72-4.16)$ & $1.99(0.83-4.77)$ & $2.76(0.92-8.28)$ & 0.064 \\
\hline Model 2 & 1.00 & $1.85(0.76-4.49)$ & $2.23(0.91-5.43)$ & $3.39(1.08-10.70)$ & 0.034 \\
\hline Model 3 & 1.00 & $1.57(0.60-4.06)$ & $2.03(0.78-5.28)$ & $3.78(1.08-13.27)$ & 0.043 \\
\hline \multicolumn{6}{|l|}{ Hypertension } \\
\hline Model 1 & 1.00 & $0.77(0.25-2.36)$ & $1.29(0.46-3.57)$ & $2.64(1.03-6.80)$ & 0.022 \\
\hline Model 2 & 1.00 & $0.80(0.26-2.44)$ & $1.36(0.48-3.84)$ & $2.84(1.04-7.19)$ & 0.021 \\
\hline Model 3 & 1.00 & $0.79(0.26-2.44)$ & $1.37(0.49-3.84)$ & $2.74(1.05-7.19)$ & 0.018 \\
\hline \multicolumn{6}{|l|}{ High FPG } \\
\hline Model 1 & 1.00 & $0.58(0.21-1.60)$ & $1.97(0.86-4.53)$ & $1.12(0.41-3.10)$ & 0.243 \\
\hline Model 2 & 1.00 & $0.56(0.20-1.56)$ & $1.95(0.84-4.54)$ & $1.11(0.40-3.11)$ & 0.255 \\
\hline Model 3 & 1.00 & $0.55(0.20-1.54)$ & $1.93(0.83-4.50)$ & $1.12(0.40-3.12)$ & 0.251 \\
\hline \multicolumn{6}{|l|}{ High TGs } \\
\hline Model 1 & 1.00 & $1.04(0.35-3.11)$ & $2.01(0.71-5.68)$ & $1.89(0.65-5.60)$ & 0.137 \\
\hline Model 2 & 1.00 & $1.03(0.34-3.12)$ & $2.04(0.71-5.89)$ & $1.97(0.46-6.02)$ & 0.124 \\
\hline Model 3 & 1.00 & $0.83(0.27-2.58)$ & $1.91(0.66-5.50)$ & $1.75(0.57-5.37)$ & 0.148 \\
\hline Fruit juice drink (ml/d) & 1.31 & 8.29 & 20.40 & 67.14 & \\
\hline \multicolumn{6}{|l|}{ MetS } \\
\hline Model 1 & 1.00 & $1.17(0.46-2.96)$ & $0.62(0.22-1.79)$ & $1.18(0.46-3.06)$ & 0.993 \\
\hline Model 2 & 1.00 & $1.10(0.42-2.82)$ & $0.60(0.20-1.78)$ & $1.17(0.42-3.16)$ & 0.984 \\
\hline Model 3 & 1.00 & $1.13(0.43-2.91)$ & $0.64(0.21-1.92)$ & $1.19(0.44-3.21)$ & 0.947 \\
\hline \multicolumn{6}{|l|}{ Low HDL-C } \\
\hline Model 1 & 1.00 & $0.76(0.27-2.14)$ & $0.76(0.29-2.01)$ & $0.49(0.16-1.49)$ & 0.235 \\
\hline Model 2 & 1.00 & $0.68(0.23-1.99)$ & $0.70(0.26-1.86)$ & $0.43(0.14-1.36)$ & 0.171 \\
\hline Model 3 & 1.00 & $0.69(0.23-2.02)$ & $0.72(0.27-1.92)$ & $0.44(0.14-1.39)$ & 0.181 \\
\hline \multicolumn{6}{|l|}{ Abdominal obesity } \\
\hline Model 1 & 1.00 & $1.54(0.71-3.35)$ & $0.75(0.31-1.80)$ & $1.32(0.59-2.95)$ & 0.899 \\
\hline Model 2 & 1.00 & $1.53(0.69-3.78)$ & $0.72(0.29-1.77)$ & $1.15(0.48-2.77)$ & 0.854 \\
\hline Model 3 & 1.00 & $2.31(0.95-5.61)$ & $0.88(0.33-2.35)$ & $1.26(0.48-3.34)$ & 0.865 \\
\hline \multicolumn{6}{|l|}{ Hypertension } \\
\hline Model 1 & 1.00 & $1.72(0.63-4.72)$ & $1.48(0.53-4.14)$ & $1.10(0.38-3.21)$ & 0.990 \\
\hline Model 2 & 1.00 & $1.86(0.67-5.19)$ & $1.65(0.57-4.77)$ & $1.18(0.39-3.58)$ & 0.878 \\
\hline Model 3 & 1.00 & $2.00(0.71-5.66)$ & $1.91(0.65-5.60)$ & $1.28(0.42-3.94)$ & 0.748 \\
\hline
\end{tabular}


Table 3 Multivariable-adjusted ORs (95\% Cls) of incident MetS and its components by quartiles of sugar sweetened carbonated soft drink and fruit juice drink intakes after 3.6 years of follow up among children and adolescents (Continued)

\begin{tabular}{|c|c|c|c|c|c|}
\hline \multicolumn{6}{|l|}{ High FPG } \\
\hline Model 1 & 1.00 & $1.17(0.46-2.97)$ & $0.62(0.22-1.79)$ & $1.18(0.46-3.06)$ & 0.993 \\
\hline Model 2 & 1.00 & $1.10(0.43-2.82)$ & $0.60(0.20-1.78)$ & $1.17(0.44-3.16)$ & 0.984 \\
\hline Model 3 & 1.00 & $1.13(0.44-2.91)$ & $0.64(0.21-1.92)$ & $1.19(0.44-3.21)$ & 0.947 \\
\hline \multicolumn{6}{|l|}{ High TGs } \\
\hline Model 1 & 1.00 & $0.62(0.23-1.63)$ & $0.49(0.18-1.31)$ & $0.79(0.30-2.07)$ & 0.464 \\
\hline Model 2 & 1.00 & $0.60(0.22-1.60)$ & $0.43(0.15-1.20)$ & $0.77(0.28-2.11)$ & 0.419 \\
\hline Model 3 & 1.00 & $0.64(0.24-1.73)$ & $0.47(0.16-1.34)$ & $0.84(0.30-2.33)$ & 0.544 \\
\hline
\end{tabular}

MetS metabolic syndrome, HDL-C high density lipoprotein-cholesterol, FPG fasting plasma glucose, TGs triglycerides

Model1: Adjusted for baseline age, sex, total energy intake, physical activity, and family history of diabetes

Model 2: Additionally adjusted for dietary fiber, tea and coffee, red and processed meat, fruit, and vegetable

Model 3: Additionally adjusted for body mass index

${ }^{1}$ Based on logistic regression model using median intake of sugar sweetened beverages in each quartile as a continuous variable

Dietary behavior, socio-economic factors, and physiological effects explain the unfavorable effects of higher SSB consumption on metabolic abnormalities [29]; subjects with increased SSB consumption may show properties of unhealthy dietary pattern [33]. In the current study, individuals with higher SSB consumption had higher intakes of total energy, trans and saturated fatty acids, fructose, simple sugars, and glycemic load. Lower socioeconomic status and increased hours of watching TV were associated with increased SSB intake; children from low socioeconomic status areas consumed sugar sweetened soft drinks approximately twice as common [34]. In the current investigation, $35 \%$ parents of children studied had education levels $<12$ years, which is an important point to consider in interpretation of findings. Several studies highlight the potential for parents to influence SSB intakes of children and adolescents, possibly as they are the main purchasers of food and beverages consumed; those who have parents or caregivers with high education status consume lower amounts of SSBs compared to those who have parents with lower levels of education [32]. Because SSBs are in liquid form, they induce lower satiety and higher energy intake, compared to solid form of foods [10]. Fruit juice drinks in Iran include both natural and synthetic fruit juices with added sugars. Compared to SSSDs and fruit juice drinks, other sugar sweetened beverages such as beverages made with powdered flavoring, non-juice fruit-flavored drinks, sports drinks, artificial non-caloric carbonated soft drinks, or sweetened coffee drinks are far less frequently consumed in Iran, they were not considered in our analysis. Higher SSB consumption has indirect and direct associations with insulin resistance and MetS; the direct association is due to the glycemic effects of the large amounts of absorbed sugars or metabolic effects of fructose and the indirect association because of weight gain. Evidence indicates that higher SSB consumption is concomitant with excess body weight gain, which may lead to unhealthy metabolic homeostasis $[35,36]$. In the current study, BMI rose according to the quartiles of SSB consumption; however, the associations between incident MetS and higher SSB intakes remained significant even after controlling for BMI, indicating that SSB may affect insulin resistance via pathways that do not include excess weight. The physiological effect of SSB consumption on metabolic abnormalities may be explained by high dietary glycemic load leading to a postprandial rise in blood glucose and insulin concentrations, which over time may lead to hyperinsulinemia and insulin resistance [37]. Also, an increase in glycemic load occurred simultaneously with exacerbated levels of inflammatory biomarkers such as interleukin-6, linked to insulin resistance [38]. Furthermore, the effects of fructose as the main constituent of additive nutritive sweeteners such as high fructose corn syrup and sucrose on metabolic profile are well defined in animal and human studies [39, 40]. High fructose diet increased lipogenesis, leptin resistance, intra-abdominal fat storage, hunger rating, energy intake, CRP concentration, blood pressure, and induced impaired fasting glucose by insulin resistance in the liver and adipose tissue $[40,41]$. Fructose is preferentially metabolized to lipid in the liver leading to increased triglyceride levels, which have been associated with development of insulin resistance [42]. Moreover, fructose increased serum uric acid and enhanced reabsorption of salt and water in the small intestine and kidney, indicating the hypertensioninduced effects of fructose [43].

Our study has both strengths and limitations. Some limitations of our analysis deserve comment; using an FFQ cannot estimate the 'actual' intake of participants. However, we used the FFQ to rank individuals based on their habitual intakes of SSBs. Also, the low-resolution capacity of FFQ that relays in memory of food consumption is critical at any age but, worst in children. However, for the children and adolescents, FFQ is an instrument, which can be administered in large populations and be accurate, reproducible, and relatively 
inexpensive for repeated measures. Several studies show that past dietary intake of children and adolescents can be measured reasonably well by FFQ [44-46]. Using well-trained interviewers who are skilled in the art of asking questions that help subjects remember what they ate and asking mothers about the type and quantity of meals and snacks when children were unable to recall may help minimizing the memory limitations. Another limitation was the lack of data on puberty stage. In addition, the fruit juice drink item in the FFQ was not assessed separately for $100 \%$ fruit juice and sugar sweetened synthetic juice drinks that are not $100 \%$ juice. Furthermore, the SSSD item assessed in the FFQ did not differentiate between the artificial non-caloric sweeteners and those containing caloric sugar e.g. fructose or sucrose. As for strengths, despite the limitations, to our knowledge, this is the first study to evaluate SSB consumption in relation to incidence of MetS after a 3.6 year follow-up among children and adolescents. Longitudinal design of the study allowed us to confirm the role of SSB consumption on the incidence of MetS. Furthermore, collection of socio-demographic information of the population-based cohort subjects facilitated a thorough investigation of possible confounders.

\section{Conclusion}

Our results examining the prospective associations provide evidence that greater SSB consumption during childhood and adolescence may have undesirable effects on cardio-metabolic risk factors, some of which may be independent of BMI. The results suggest that SSB particularly SSSD consumption should be limited during early years to prevent future occurrence of MetS. Although SSBs are a disposable component of world's modern diet, findings of the current study and those from systematic reviews on the contribution of SSBs on health provide strong evidence of harm. We should not wait for absolute proof and healthy alternatives to SSBs should be promoted.

\section{Abbreviations \\ MetS: Metabolic syndrome; SSB: Sugar sweetened beverage; TLGS: Tehran Lipid and Glucose Study; TG: Triglyceride; HDL-C: High density lipoprotein cholesterol; FPG: Fasting plasma glucose; FFQ: Food frequency questionnaire; USDA: United States department of agriculture; SSSD: Ssugar sweetened carbonated soft drink; DR: Dietary recall; MAQ: Modifiable activity questionnaire; MET: Metabolic equivalent task; BMI: Body mass index;} WC: Waist circumference; OR: Odds ratio; Cl: Confidence interval.

\section{Competing interests}

The authors declare that they have no competing of interests.

\section{Authors' contribution}

The project was designed and implemented by P.M and G.A. Data were analyzed and interpreted by E.Y and S.H-N. P.M, E.Y, G.A and S.H-N prepared the manuscript. P.M and F.A supervised overall project. All authors read and approved the final version of manuscript.

\section{Acknowledgment}

This work was funded by a grant from the Research Institute for Endocrine Sciences, Shahid Beheshti University of Medical Sciences, Tehran, Iran. The authors express appreciation to the participants in the Tehran Lipid and Glucose Study for their enthusiastic support, and the staff of the Tehran Lipid and Glucose Study Unit of the Research Institute for Endocrine Sciences, for their valuable help. The authors wish to acknowledge Ms Niloofar Shiva for critical editing of English grammar and syntax of the manuscript.

\section{Author details}

'Nutrition and Endocrine Research Center, Research Institute for Endocrine Sciences, Shahid Beheshti University of Medical Sciences, Tehran, Iran. ${ }^{2}$ Endocrine Research Center, Research Institute for Endocrine Sciences, Shahid Beheshti University of Medical Sciences, Tehran, Iran.

Received: 6 April 2015 Accepted: 13 July 2015

Published online: 30 July 2015

\section{References}

1. Friend A, Craig L, Turner S. The prevalence of metabolic syndrome in children: a systematic review of the literature. Metab Syndr Relat Disord. 2013;11:71-80.

2. Morrison JA, Friedman LA, Wang P, Glueck CJ. Metabolic syndrome in childhood predicts adult metabolic syndrome and type 2 diabetes mellitus 25 to 30 years later. J Pediatr. 2008;152:201-6.

3. Morrison JA, Friedman LA, Gray-McGuire C. Metabolic syndrome in childhood predicts adult cardiovascular disease 25 years later: the Princeton Lipid Research Clinics Follow-up Study. Pediatrics. 2007;120:340-5.

4. Lusis AJ, Attie AD, Reue K. Metabolic syndrome: from epidemiology to systems biology. Nat Rev Genet. 2008;9:819-30.

5. Yudkin JS. Insulin resistance and the metabolic syndrome-or the pitfalls of epidemiology. Diabetologia. 2007;50:1576-86.

6. Weiss R, Bremer AA, Lustig RH. What is metabolic syndrome, and why are children getting it? Ann N Y Acad Sci. 2013;1281:123-40.

7. Harrington $\mathrm{S}$. The role of sugar-sweetened beverage consumption in adolescent obesity: a review of the literature. J Sch Nurs. 2008;24:3-12.

8. Overby NC, Lillegaard IT, Johansson L, Andersen LF. High intake of added sugar among Norwegian children and adolescents. Public Health Nutr. 2004;7:285-93.

9. Reedy J, Krebs-Smith SM. Dietary sources of energy, solid fats, and added sugars among children and adolescents in the United States. J Am Diet Assoc. 2010;110:1477-84.

10. Malik VS, Popkin BM, Bray GA, Despres JP, Hu FB. Sugar-sweetened beverages, obesity, type 2 diabetes mellitus, and cardiovascular disease risk. Circulation. 2010;121:1356-64

11. Malik VS, Pan A, Willett WC, Hu FB. Sugar-sweetened beverages and weight gain in children and adults: a systematic review and meta-analysis. Am J Clin Nutr. 2013;98:1084-102.

12. Althuis MD, Weed DL. Evidence mapping: methodologic foundations and application to intervention and observational research on sugar-sweetened beverages and health outcomes. Am J Clin Nutr. 2013;98:755-68.

13. Chan TF, Lin WT, Huang HL, Lee CY, Wu PW, Chiu YW, et al. Consumption of sugar-sweetened beverages is associated with components of the metabolic syndrome in adolescents. Nutrients. 2014;6:2088-103.

14. Azizi F, Ghanbarian A, Momenan AA, Hadaegh F, Mirmiran P, Hedayati M, et al. Prevention of non-communicable disease in a population in nutrition transition: Tehran Lipid and Glucose Study phase II. Trials. 2009;10:5.

15. Asghari G, Mirmiran P, Rashidkhani B, Asghari-Jafarabadi M, Mehran M, Azizi F. The association between diet quality indices and obesity: Tehran Lipid and Glucose Study. Arch Iran Med. 2012;15:599-605.

16. Mirmiran P, Esfahani FH, Mehrabi Y, Hedayati M, Azizi F. Reliability and relative validity of an FFQ for nutrients in the Tehran lipid and glucose study. Public Health Nutr. 2010;13:654-62.

17. Esfahani FH, Asghari G, Mirmiran P, Azizi F. Reproducibility and relative validity of food group intake in a food frequency questionnaire developed for the Tehran Lipid and Glucose Study. J Epidemiol. 2010;20:150-8.

18. Asghari G, Rezazadeh A, Hosseini-Esfahani F, Mehrabi Y, Mirmiran P, Azizi F. Reliability, comparative validity and stability of dietary patterns derived from an FFQ in the Tehran Lipid and Glucose Study. Br J Nutr. 2012;108:1109-17.

19. Ghaffarpour M, Houshiar-Rad A, Kianfar H. The manual for household measures, cooking yields factors and edible portion of food. Tehran: Keshaverzi press; 1999. 
20. Kriska AM, Edelstein SL, Hamman RF, Otto A, Bray GA, Mayer-Davis EJ, et al. Physical activity in individuals at risk for diabetes: Diabetes Prevention Program. Med Sci Sports Exerc. 2006;38:826-32.

21. Delshad M, Ghanbarian A, Ghaleh NR, Amirshekari G, Askari S, Azizi F. Reliability and validity of the modifiable activity questionnaire for an Iranian urban adolescent population. Int J Prev Med. 2015;6:3.

22. Cook $S$, Weitzman $M$, Auinger $P$, Nguyen $M$, Dietz WH. Prevalence of a metabolic syndrome phenotype in adolescents: findings from the third National Health and Nutrition Examination Survey, 1988-1994. Arch Pediatr Adolesc Med. 2003;157:821-7.

23. Kelishadi R, Gouya MM, Ardalan G, Hosseini M, Motaghian M, Delavari A, et al. First reference curves of waist and hip circumferences in an Asian population of youths: CASPIAN study. J Trop Pediatr. 2007;53:158-64.

24. National High Blood Pressure Education Program Working Group on High Blood Pressure in Children and adolescent. The fourth report on the diagnosis, evaluation, and treatment of high blood pressure in children and adolescents. Pediatrics. 2004;114:555-76.

25. Genuth S, Alberti KG, Bennett P, Buse J, Defronzo R, Kahn R, et al. Follow-up report on the diagnosis of diabetes mellitus. Diabetes Care. 2003;26:3160-7.

26. Alberti KG, Eckel RH, Grundy SM, Zimmet PZ, Cleeman Jl, Donato KA, et al. Harmonizing the metabolic syndrome: a joint interim statement of the International Diabetes Federation Task Force on Epidemiology and Prevention; National Heart, Lung, and Blood Institute; American Heart Association; World Heart Federation; International Atherosclerosis Society; and International Association for the Study of Obesity. Circulation. 2009;120:1640-5.

27. Delavari A, Forouzanfar MH, Alikhani S, Sharifian A, Kelishadi R. First nationwide study of the prevalence of the metabolic syndrome and optimal cutoff points of waist circumference in the Middle East: the national survey of risk factors for noncommunicable diseases of Iran. Diabetes Care. 2009;32:1092-7.

28. Barrio-Lopez MT, Martinez-Gonzalez MA, Fernandez-Montero A, Beunza JJ, Zazpe I, Bes-Rastrollo M. Prospective study of changes in sugar-sweetened beverage consumption and the incidence of the metabolic syndrome and its components: the SUN cohort. Br J Nutr. 2013;110:1722-31.

29. Dhingra R, Sullivan L, Jacques PF, Wang TJ, Fox CS, Meigs JB, et al. Soft drink consumption and risk of developing cardiometabolic risk factors and the metabolic syndrome in middle-aged adults in the community. Circulation. 2007;116:480-8.

30. Wang JW, Mark S, Henderson M, O'Loughlin J, Tremblay A, Wortman J, et al. Adiposity and glucose intolerance exacerbate components of metabolic syndrome in children consuming sugar-sweetened beverages: QUALITY cohort study. Pediatr Obes. 2013;8:284-93.

31. Bremer AA, Auinger $P$, Byrd RS. Relationship between insulin resistanceassociated metabolic parameters and anthropometric measurements with sugar-sweetened beverage intake and physical activity levels in US adolescents: findings from the 1999-2004 National Health and Nutrition Examination Survey. Arch Pediatr Adolesc Med. 2009;163:328-35.

32. Ambrosini GL, Oddy WH, Huang RC, Mori TA, Beilin $L$, Jebb SA. Prospective associations between sugar-sweetened beverage intakes and cardiometabolic risk factors in adolescents. Am J Clin Nutr. 2013;98:327-34.

33. Shang L, O'Loughlin J, Tremblay A, Gray-Donald K. The association between food patterns and adiposity among Canadian children at risk of overweight. Appl Physiol Nutr Metab. 2014;39:195-201.

34. Clifton PM, Chan L, Moss CL, Miller MD, Cobiac L. Beverage intake and obesity in Australian children. Nutr Metab (Lond). 2011;8:87.

35. Bray GA, Popkin BM. Dietary sugar and body weight: have we reached a crisis in the epidemic of obesity and diabetes?: health be damned! Pour on the sugar. Diabetes Care. 2014;37:950-6.

36. Wang J. Consumption of added sugars and development of metabolic syndrome components among a sample of youth at risk of obesity. Appl Physiol Nutr Metab. 2014;39:512.

37. Dominguez Coello S, Cabrera De Leon A, Rodriguez Perez MC, Borges Alamo C, Carrillo Fernandez L, Almeida Gonzalez D, et al. Association between glycemic index, glycemic load, and fructose with insulin resistance: the CDC of the Canary Islands study. Eur J Nutr. 2010;49:505-12.

38. Goletzke J, Buyken AE, Joslowski G, Bolzenius K, Remer T, Carstensen M, et al. Increased intake of carbohydrates from sources with a higher glycemic index and lower consumption of whole grains during puberty are prospectively associated with higher IL-6 concentrations in younger adulthood among healthy individuals. J Nutr. 2014;144:1586-93.
39. Kelishadi R, Mansourian M, Heidari-Beni M. Association of fructose consumption and components of metabolic syndrome in human studies: a systematic review and meta-analysis. Nutrition. 2014;30:503-10.

40. Stanhope KL, Schwarz JM, Havel PJ. Adverse metabolic effects of dietary fructose: results from the recent epidemiological, clinical, and mechanistic studies. Curr Opin Lipidol. 2013;24:198-206.

41. Hosseini-Esfahani F, Bahadoran Z, Mirmiran P, Hosseinpour-Niazi S, Hosseinpanah F, Azizi F. Dietary fructose and risk of metabolic syndrome in adults: Tehran Lipid and Glucose study. Nutr Metab (Lond). 2011;8:50.

42. Basciano H, Federico L, Adeli K. Fructose, insulin resistance, and metabolic dyslipidemia. Nutr Metab (Lond). 2005;2:5

43. Bobridge KS, Haines GL, Mori TA, Beilin LJ, Oddy WH, Sherriff J, et al. Dietary fructose in relation to blood pressure and serum uric acid in adolescent boys and girls. J Hum Hypertens. 2013;27:217-24.

44. Eck LH, Klesges RC, Hanson CL, Slawson D, Portis L, Lavasque ME. Measuring short-term dietary intake: development and testing of a 1-week food frequency questionnaire. J Am Diet Assoc. 1991;91:940-5.

45. Blom L, Lundmark K, Dahlquist G, Persson LA. Estimating children's eating habits. Validity of a questionnaire measuring food frequency compared to a 7-day record. Acta Paediatr Scand. 1989;78:858-64.

46. Frank GC, Nicklas TA, Webber LS, Major C, Miller JF, Berenson GS. A food frequency questionnaire for adolescents: defining eating patterns. J Am Diet Assoc. 1992;92:313-8.

\section{Submit your next manuscript to BioMed Central and take full advantage of:}

- Convenient online submission

- Thorough peer review

- No space constraints or color figure charges

- Immediate publication on acceptance

- Inclusion in PubMed, CAS, Scopus and Google Scholar

- Research which is freely available for redistribution 